\title{
Periprocedural Risk of Stroke Is Elevated in Patients with End-Stage Renal Disease on Hemodialysis
}

\author{
Laurel Cherian James Conners Shawna Cutting Vivien H. Lee \\ Sarah Song
}

Section of Cerebrovascular Disease, Department of Neurology, Rush University Medical

Center, Chicago, Ill., USA

\section{Key Words}

Ischemic stroke $\cdot$ Renal disease $\cdot$ Hemodialysis

\begin{abstract}
Objective: To describe the most common clinical factors and stroke etiologies in a case series of patients with end-stage renal disease on hemodialysis (ESRD/HD) with transient ischemic attack (TIA) or ischemic stroke (IS). Background: Prior studies have shown that patients on $\mathrm{HD}$ are at an elevated risk of stroke, but these studies have focused on the overall stroke risk. This case series sought to determine the percentage of acute ischemic events that occur during or immediately after HD. Methods: ICD-9 codes were used to identify IS and TIA patients with ESRD/HD admitted to the stroke service from August 22, 2011, to June 21, 2014. Charts were reviewed to determine the age, sex, and race/ethnicity of the cohort. TIA/IS diagnosis was confirmed by a vascular neurologist. Clinical factors were assessed, including: onset during or shortly after HD, defined as occurring within $12 \mathrm{~h}$ of $\mathrm{HD}$; the presence of a lesion on diffusion-weighted MRI; hypotension, hyponatremia, or hypoglycemia at symptom onset; the stroke etiology; the presence of focal neurologic deficits; whether the patient was in the window period for intravenous tissue plasminogen activator (IVtPA) upon presentation, and whether the patient received IVtPA. Results: We identified 34 ESRD/HD patients with a diagnosis of TIA/stroke in the specified time period. A majority of patients (70.6\%) were African American. Patient age ranged from 32 to 84 years, with a median age of 67 years. Twentyseven patients (79.4\%) had confirmed ischemic infarcts on diffusion-weighted MRI. Seven patients (20.6\%) were diagnosed with TIA. In 13 patients (38.2\%), symptom onset occurred during or shortly after HD. Of these 13 patients, 8 (61.5\%) had symptom onset during HD. Three patients (8.8\%) had documented hypotension near the time of symptom onset, and 2 (5.9\%) were hyponatremic on presentation to the emergency department. The distribution of
\end{abstract}



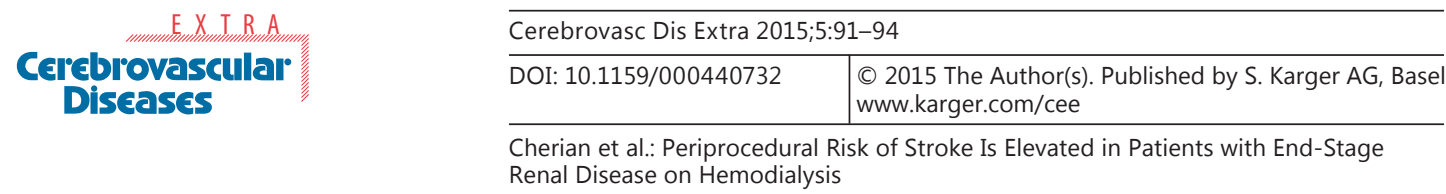

stroke etiologies was as follows: 4 (11.8\%) watershed distribution, 1 (2.9\%) large artery atherosclerosis, 2 (20.6\%) small vessel disease, 10 (29.4\%) cardioembolic, and 9 (26.5\%) cryptogenic. In 28 patients (82.4\%), focal neurologic deficits were observed on presentation. Nine patients (26.5\%) arrived within the window period for IVtPA, and $4(11.8 \%)$ were eligible and received IVtPA. Conclusions: Of all patients with ESRD on HD admitted to the stroke service over the study period, over one third (38.3\%) had the onset of their ischemic event during or shortly after HD, and nearly one quarter (23.5\%) had the onset during HD. While clinicians may be tempted to attribute neurologic changes after HD to metabolic etiologies, they should also be aware that HD represents a period of elevated risk for acute ischemia.

(C) 2015 The Author(s)

Published by S. Karger AG, Basel

\section{Introduction}

North America experienced a five-fold increase in the number of patients with end-stage renal disease (ESRD) between 1980 and 2001 [1]. Prior studies have shown that patients on hemodialysis (HD) are at an elevated risk of stroke, with the risk increasing as renal function declines [2, 3]. The US National Health and Nutrition Examination Survey (NHANES) 19992004 showed that patients with chronic kidney disease had a five-fold increase in stroke risk [3]. Despite these statistics, patients with a history of cardiovascular disease and ESRD/HD may be undertreated with aspirin, despite having no identifiable contraindication to it [2].

HD can also lead to the development of a hypercoagulable state, in which prothrombin fragments $1+2$ and thrombin-antithrombin complexes are elevated, while levels of endogenous anticoagulants, such as plasminogen, tissue plasminogen activator, proteins $\mathrm{C}$ and $\mathrm{S}$, and antithrombin are decreased [1]. Routine periods of immobility several times a week may also be a factor predisposing to thrombotic events, as may endothelial trauma related to catheter placement or malfunction, and the use of erythropoietin [1].

Additionally, HD has been associated with cardiac structural modifications and is also known to cause shifts in electrolyte balances, both of which may put patients at a higher risk of atrial fibrillation. Studies have shown that patient age, duration of HD therapy, and left atrial dilatation are associated with atrial fibrillation [4]. Atrial fibrillation has been reported in up to $13-27 \%$ of patients, which is much higher than in the general population $[1,4]$. In a study evaluating the etiologies of ischemic stroke (IS) in ESRD/HD patients using the Trial of Org 10172 in Acute Stroke Treatment (TOAST) criteria, cardioembolism accounted for 28\% of ischemic events [5]. Clinicians may be more hesitant to anticoagulate ESRD/HD patients, however, due to an increased risk of bleeding $[1,6]$.

While there is extensive evidence showing an increased stroke risk in ESRD/HD patients, which increases over the months to years of HD therapy, there is little to no available data on the acute onset of stroke symptoms with regard to the most recent HD session. This case series sought to determine the percentage of acute ischemic events that occur during or immediately after HD and to describe the most common clinical factors and stroke etiologies in these patients.

\section{Methods}

ICD-9 codes were used to identify IS and transient ischemic attack (TIA) patients with ESRD/HD admitted to the stroke service from August 22, 2011, to June 21, 2014. Charts were reviewed to determine the age, sex, and race/ethnicity of the cohort. TIA/IS diagnosis was 
Cherian et al.: Periprocedural Risk of Stroke Is Elevated in Patients with End-Stage

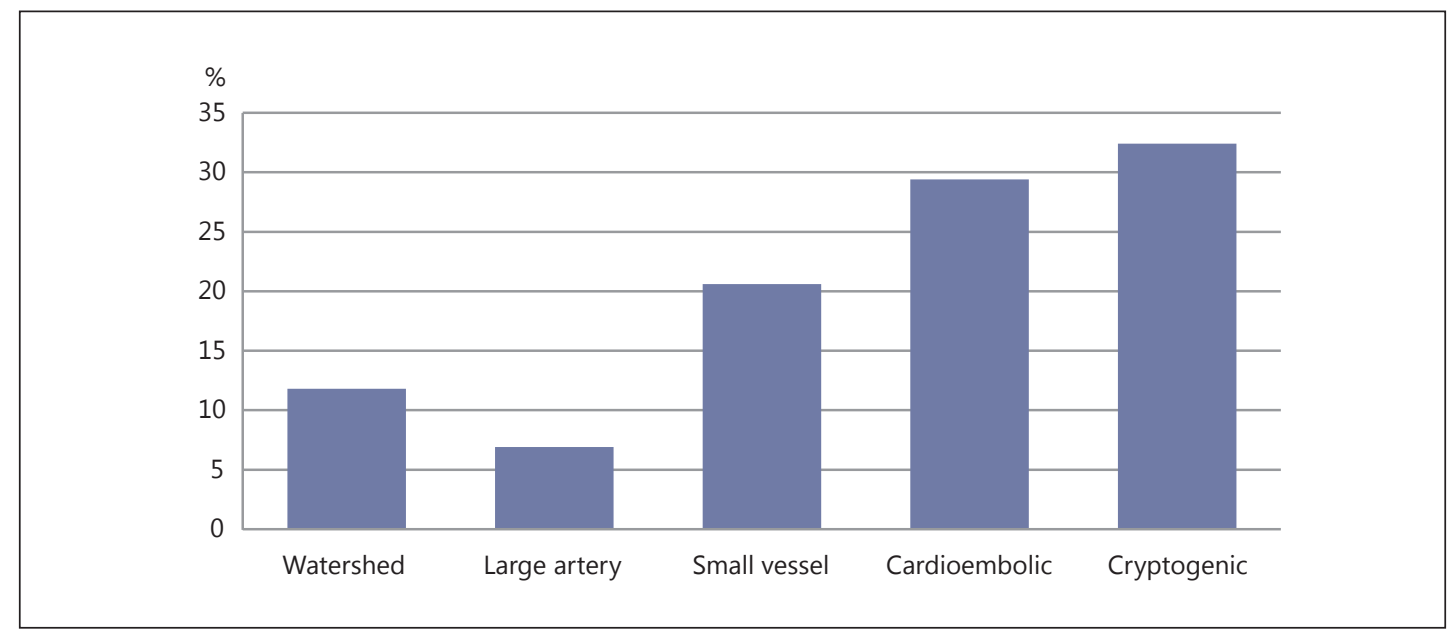

Fig. 1. Etiology of ischemic events in HD patients.

Fig. 2. Periprocedural onset of ischemic events in HD patients.

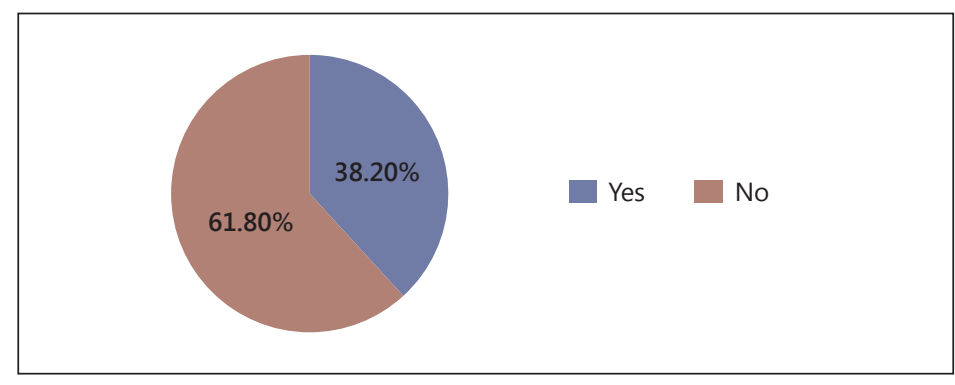

confirmed by a vascular neurologist at the time of the event. Clinical factors were assessed, including: onset during or shortly after HD, defined as $0-12 \mathrm{~h}$ from HD; the presence of a lesion on diffusion-weighted MRI; hypotension, hyponatremia, or hypoglycemia at symptom onset; the stroke etiology; the presence of focal neurologic deficits; whether the patient was in the window period for intravenous tissue plasminogen activator (IVtPA) upon presentation, and whether the patient received IVtPA.

\section{Results}

We identified 34 ESRD/HD patients with a diagnosis of TIA/stroke in the specified time period. A majority of patients (70.6\%) were African American. Patient age ranged from 32 to 84 years, with a median age of 67 years. Twenty-seven patients $(79.4 \%)$ had confirmed ischemic infarcts on diffusion-weighted MRI. Seven patients (20.6\%) were diagnosed with TIA. In 13 patients (38.2\%), symptom onset occurred during or shortly after HD, with 8 patients $(23.5 \%)$ having symptom onset during HD, $3(8.8 \%)$ immediately after HD, 1 (2.9\%) within $3 \mathrm{~h}$ from HD, and 1 (2.9\%) within $12 \mathrm{~h}$ of HD. Three patients (8.8\%) had documented hypotension near the time of symptom onset, and 2 (5.9\%) were hyponatremic on presentation to the emergency department. The distribution of stroke etiologies was as follows (fig. 1): 4 (11.8\%) watershed distribution, 1 (2.9\%) large artery atherosclerosis, 2 (20.6\%) small vessel disease, 10 (29.4\%) cardioembolic, and 9 (26.5\%) cryptogenic. In 28 
patients (82.4\%), focal neurologic deficits were observed on presentation. Nine patients $(26.5 \%)$ arrived within the window period for IVtPA, and $4(11.8 \%)$ were eligible and received IVtPA.

\section{Discussion}

Patients with ESRD/HD have a significantly elevated risk of stroke, which may be acutely elevated during and immediately after HD, as our data suggest. The stroke etiologies were similar to a prior study on stroke in patients with ESRD/HD, which also showed cardioembolism (28\%) and cryptogenic/unknown (23\%) as the most common etiologies [5]. Rates of atrial fibrillation tend to be higher in this population, but patients may be less likely to be treated with prophylactic anticoagulation due to concerns about a higher risk of bleeding complications $[1,6,7]$, and this may explain the high prevalence of cardioembolic events. IS consistent with a watershed distribution occurred in $11.8 \%$ of patients, which was close to the percentage (8.8\%) of patients who had documented hypotension near the time of their event. Of all patients with ESRD on HD admitted to the stroke service over the study period, over one third (38.3\%) had the onset of their ischemic event during or shortly after HD (fig. 2), and the majority of these patients (8 of 13) had symptom onset during HD. Clinicians should be aware that ESRD/HD patients are at a high risk for stroke, and this risk may be acutely elevated during HD. ESRD/HD patients may especially benefit from stroke education, as they are in a health care setting during HD and could feasibly get prompt treatment if they and their caregivers are able to recognize the signs/symptoms of stroke. In conclusion, the periprocedural stroke risk appears to be elevated in ESRD/HD patients.

\section{References}

1 Lo DS, Rabbat CG, Clase CM: Thromboembolism and anticoagulant management in hemodialysis patients: a practical guide to clinical management. Thromb Res 2006;118:385-395.

2 Dempster DW, Rosenstock JL, Schwimmer JA, Panagopoulos G, DeVita MV, Michelis MF: Underutilization of aspirin in hemodialysis patients for primary and secondary prevention of cardiovascular disease. Clin Nephrol 2005;64:371-377.

3 Muntner P, Judd SE, McClellan W, Meschia JF, Warnock DG, Howard VJ: Incidence of stroke symptoms among adults with chronic kidney disease: results from the REasons for Geographic And Racial Differences in Stroke (REGARDS) study. Nephrol Dial Transplant 2012;27:166-173.

4 Genovesi S, Pogliani D, Faini A, Valsecchi MG, Riva A, Stefani F, et al: Prevalence of atrial fibrillation and associated factors in a population of long-term hemodialysis patients. Am J Kidney Dis 2005;46:897-902.

5 Sozio SM, Armstrong PA, Coresh J, Jaar BG, Fink NE, Plantinga LC, et al: Cerebrovascular disease incidence, characteristics, and outcomes in patients initiating dialysis: the choices for healthy outcomes in caring for ESRD (CHOICE) study. Am J Kidney Dis 2009;54:468-477.

6 Wetmore JB, Ellerbeck EF, Mahnken JD, Phadnis M, Rigler SK, Mukhopadhyay P, et al: Atrial fibrillation and risk of stroke in dialysis patients. Ann Epidemiol 2013;23:112-118.

7 Marsh EB, Llinas RH, Hillis AE, Gottesman RF: Hemorrhagic transformation in patients with acute ischaemic stroke and an indication for anticoagulation. Eur J Neurol 2013;20:962-967. 